\title{
VC funding hits high in Q1
}

\section{Stacy Lawrence}

Last quarter, biotech stock performance remained lackluster and financing in the sector ( $\sim 12$ billion) lagged behind the first and fourth quarters of 2006. Venture capital funding, however, reached a new benchmark, surpassing $\$ 1.9$ billion—up by half in the US; Asia

\section{Biotech stock market performance}

Biotech indices continued to perform poorly in first quarter.

W Swiss exchange Dow Jones $\quad$ S\&P 500 Nasdaq BioCentury 100 Nasdaq Biotech

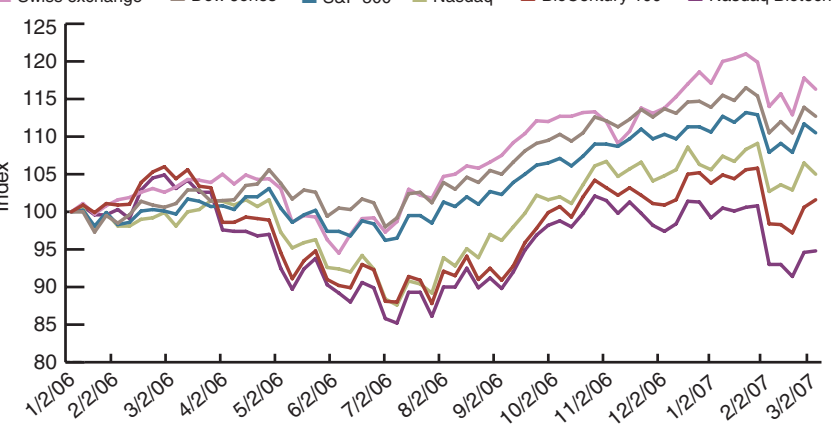

Source: Multex, BioCentury

\section{Global biotech industry financing}

In total, biotech financing in 1Q07 was off by almost one-third from 1Q07, but VC funding was plentiful.

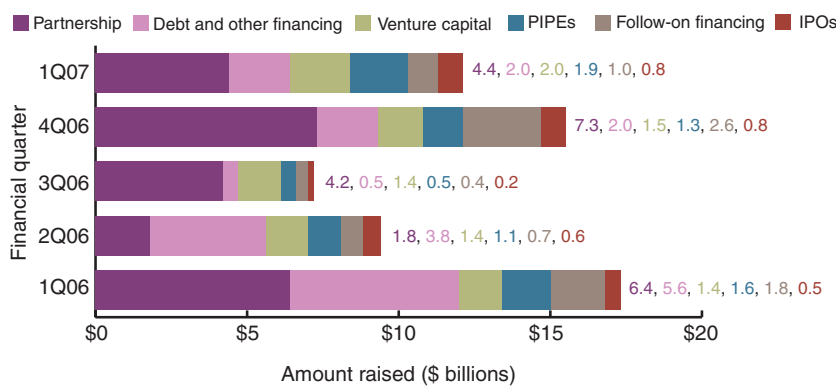

also witnessed an uptick, whereas Europe performed poorly. The amount raised in initial public offerings raising (IPOs) held steady, with European IPOs raising the most funds for the second successive quarter.

\section{Global biotech initial public offerings}

European IPOs raised more than those in North America for the second consecutive quarter; IPOs in the Far East were also buoyant.

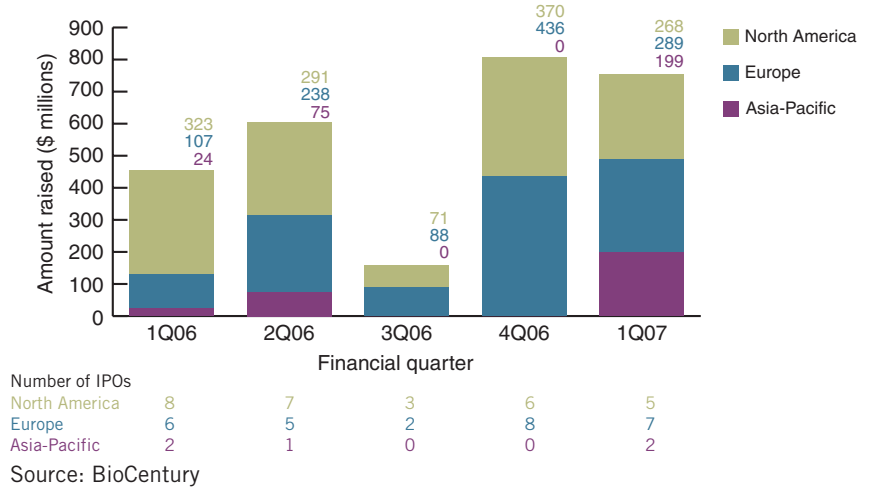

\section{Global biotech venture capital investment}

Biotech venture investing in $Q 1$ reached an all-time high ( $\$ 1.9$ billion), surpassing the previous high of $\$ 1.7$ billion in $4 \mathrm{Q} 00$.

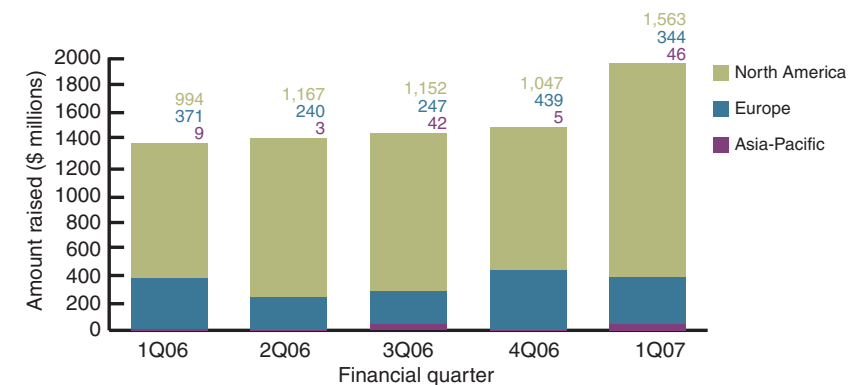

Source: BioCentury

\begin{tabular}{|c|c|c|c|c|}
\hline IPOs & Company (Lead underwriters) & Amount raised ( $\$$ millions) & Percent change in stock price since offer ${ }^{1}$ & Date launched \\
\hline & 3SBIO (UBS) & 115 & $-31 \%$ & 7-Feb \\
\hline & Tongjitang Chinese Medicines Co.(Merrill Lynch and UBS) & 84 & $1 \%$ & 16-Mar \\
\hline & Molecular Insight (RBC Capital Markets and Jefferies \& Co.) & 70 & $-15 \%$ & 2-Feb \\
\hline & Tigenix (ING) & 53 & $36 \%$ & 21-Mar \\
\hline & Synta (Bear Stearns and Lehman Brothers) & 50 & $-19 \%$ & 6-Feb \\
\hline \multirow[t]{6}{*}{ Venture capital } & Company Lead investors) & Amount invested (\$ millions) & Round number & Date closed \\
\hline & Targanta Therapeutics & 70 & 3 & 2-Feb \\
\hline & Novexel & 66 & 2 & 8-Jan \\
\hline & Movetis & 65 & 1 & 7-Jan \\
\hline & Ception Therapeutics & 63 & 3 & 30-Jan \\
\hline & Omeros & 63 & 5 & $12-\mathrm{Feb}$ \\
\hline \multirow[t]{6}{*}{ Mergers and acquisitions } & Target & Acquirer & Value $(\$ \text { millions })^{2}$ & Date announced \\
\hline & Biosite & Beckman Coulter & 1,550 & 26-Mar \\
\hline & MDS Diagnostics & Borealis Infrastructure & 1,142 & $26-\mathrm{Feb}$ \\
\hline & INO Therapeutics & Ikaria & 670 & $22-\mathrm{Feb}$ \\
\hline & Molecular Devices & MDS & 615 & 29-Jan \\
\hline & Adeza Biomedical & Cytyc & 452 & $12-\mathrm{Feb}$ \\
\hline \multirow[t]{6}{*}{ Licensing/ collaboration } & Researcher & Investor & Value ( $\$$ millions) & Deal description \\
\hline & Seattle Genetics & Genentech & 860 & Collaboration and development \\
\hline & Cytokinetics & Amgen & 725 & License \\
\hline & XenoPort & GlaxoSmithKline & 640 & Collaboration and development \\
\hline & Anacor & Schering-Plough & 615 & License \\
\hline & Argenta Discovery & AstraZeneca & 500 & License \\
\hline
\end{tabular}

